\title{
Utility of dermoscopy for demarcation of surgical margins in Mohs micrographic surgery*
}

\author{
Heliane Sanae Suzuki ${ }^{1}$
}

\author{
Sérgio Zuñeda Serafini ${ }^{2}$
}

\author{
Maurício Shigueru Sato ${ }^{3}$
}

\begin{abstract}
BACKGROUND: Skin cancer is the most common malignancy and can be treated in various ways. One treatment modality is Mohs micrographic surgery. Due to the increasing incidence of skin cancer in the last decades, there is a need for improvement of the Mohs technique to optimize its effectiveness. OBJестіvE: The aim of this study was to evaluate the use of dermoscopy to guide demarcation of Mohs micrographic surgery margins and ascertain whether this method can reduce operative time and, therefore, reduce surgical morbidity and cost. METHODS AND MATERIALS: The sample comprised 44 patients who underwent Mohs micrographic surgery, allocated into two groups: the control group and the intervention group. In the latter, surgical margins were guided by dermoscopy. RESULTS: There were no statistically significant differences between the two groups by chi-square analysis $(p=0.399)$. CONCLUSION: Although outcomes were similar in the two groups, demonstrating that dermoscopy does not help in the demarcation of surgical margins for Mohs micrographic surgery, the study provides a practical proposal for improvement of the Mohs technique.
\end{abstract}

Keywords: Dermoscopy; Mohs surgery; Skin neoplasms

\section{INTRODUCTION}

Skin cancer is the most common malignancy, accounting for $25 \%$ of all cases reported in Brazil. The most common forms are basal cell carcinoma (BCC), spindle cell carcinoma (SCC), and melanoma, which account for $70 \%, 25 \%$, and $4 \%$ of all skin cancers respectively. ${ }^{1}$

The estimated countrywide incidence of nonmelanoma skin cancers in 2010 was 53,410 new cases in men and 60,440 new cases in women, according to the Cancer Incidence Estimates published by the Brazilian National Cancer Institute (INCA). These figures correspond to an estimated risk of 56 new cases per 100,000 men and 61 new cases per 100,000 women. With early diagnosis and treatment, these malignancies are highly curable. Despite their high incidence, only 1,296 deaths attributable to nonmelanoma skin cancer were recorded in Brazil in $2007 .{ }^{1}$
A variety of treatment modalities are available for cutaneous neoplasms, including cryotherapy, curettage and electrodesiccation, photodynamic therapy, radiation therapy, chemotherapy, imiquimod, and surgical excision. ${ }^{2}$

Another treatment option is Mohs micrographic surgery (MMS), a particular type of dermatologic surgery that involves minimal margins controlled by performing horizontal frozen sections during the procedure. ${ }^{3,4,5,6} \mathrm{In}$ view of the increased incidence of skin cancer in the last decades, there is a need for improvement of the Mohs technique so as to optimize its efficacy., ${ }^{3,4,7,8,10,11}$

Dermoscopy, or dermatoscopy, is a noninvasive diagnostic technique that is very useful in the assessment of cutaneous lesions, as it enables visualization of structures invisible to the naked eye..$^{5,7,8,12,13,14,15}$ Dermoscopy is performed with an instrument known as a dermoscope or dermatoscope,

Received on 04.01.2013.

Approved by the Advisory Board and accepted for publication on 18.02.2013.

* Study carried out at the Dermatology Service, Teaching Hospital - Federal University of Paraná (Universidade Federal do Paraná -HC / UFPR) - Curitiba (PR), Brazil.

Financial Support: None

Conflict of Interest: None.

Board-certified dermatologist, Brazilian Society of Dermatology , Physician in private practice, Curitiba (PR), Brazil

Board-certified dermatologist, Brazilian Society of Dermatology - Professor, Department of Dermatology, Hospital de Clínicas - Universidade Federal do Paraná (HC UFPR ) - Curitiba (PR), Brazil

Board-certified dermatologist, Brazilian Society of Dermatology - Staff physician, Outpatient Mohs Surgery Clinic - Hospital de Clínicas - Universidade Federal do Paraná (HC UFPR) - Curitiba (PR), Brazil. 
which provides (on average) a tenfold magnification of skin structures. ${ }^{7,12,14}$

The present study advances a new proposal for use of dermoscopy as an adjunct to therapy rather than as a diagnostic method alone.

\section{Objective}

To assess the impact of dermoscopy on demarcation of surgical margins for MMS and ascertain whether the use of this method can shorten operative time and, consequently, decrease surgical morbidity and cost.

\section{MATERIALS AND METHODS}

All methodological aspects of this study were in compliance with the current Brazilian legislation for human subjects research, as set forth in National Health Council Resolution 196/96, and with the latest revision of the Declaration of Helsinki. The study was approved by the Research Ethics Committee of the hospital where it was conducted.

This was a prospective, non-randomized study.

Patients with nonmelanoma skin cancer and clinical indications for MMS were recruited from the outpatient oncologic dermatology clinic of Hospital de Clínicas da Universidade Federal do Paraná from April 2009 to August 2011. These patients were allocated into two groups: control (group I) and intervention - MMS with dermoscopy-guided margins (group II).

In both groups, all lesions were first assessed with the naked eye and had their margins demarcated in blue or black marker (Figure 1). In the intervention group, tumors were also assessed under dermoscopy for determination of dermoscopic margins, which were drawn in red (Figure 2). The outermost margin was used to define the surgical margin (initially $2 \mathrm{~mm}$ ).

Changes in skin texture due to tumor proliferation and a finding of tumor structures in the skin adjacent to the demarcated clinical margin were considered dermoscopic patterns indicative of lesion extension beyond the clinical margins. ${ }^{16}$ The presence of whitish or erythematous areas or superficial ulcerations on dermoscopy were deemed criteria for alter-

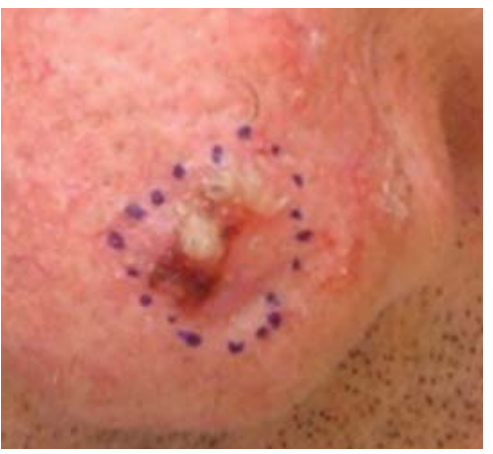

Figure 1:

Demarcation of surgical margins in a control group patient

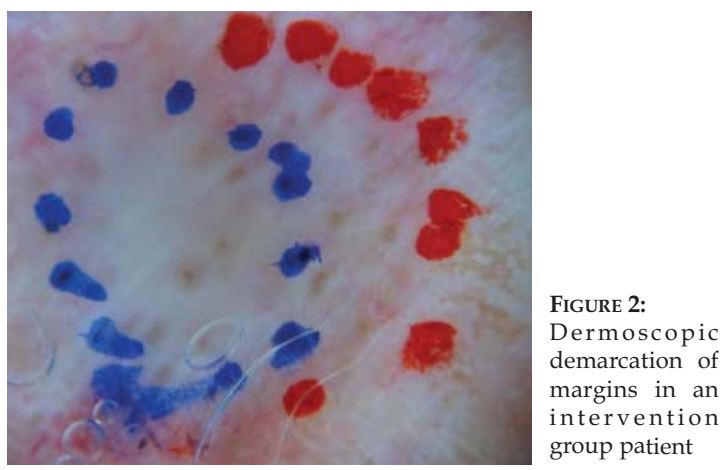

ation of the initially demarcated margins..$^{8,9,13}$ Nonmelanoma pigmented lesions were examined for structures such as blue/gray globules, leaf-like areas, and spoke-wheel areas, among other characteristics described in greater detail later. ${ }^{9}$ Examination of nonpigmented lesions was guided by the vascular structures usually present in skin lesions, such as arborizing or glomerular vessels. In case of doubt, the margins were defined on the basis of this area. ${ }^{13,15}$

The dermoscope used for the study was a DermLite Hybrid contact dermoscopy device with 10x magnification capabilities. Clinical and dermoscopic images were obtained with a 10.1-megapixel Sony DSC-W179 digital camera.

The number of surgical stages required in each of the two groups was analyzed to ascertain whether dermoscopy had an impact on the MMS workflow. Statistical analysis was conducted by means of the chisquare test, at a significance level of $5 \%$.

Any modifications to surgical margins made after dermoscopy were measured in millimeters. Furthermore, the profile of the study population including variables such as sex, age, and lesion site and histological type - was also analyzed.

\section{RESULTS}

A total of 44 patients undergoing MMS were selected for the study. The first 21 patients referred for surgical treatment were allocated to the control group, and the rest to the intervention group (dermoscopyguided surgical margins).

In the control group, the mean age was 61 years, and $62 \%$ of patients $(n=13)$ were female. In the intervention group, mean age was 58 years and $61 \%$ $(n=14)$ were female. Fourteen patients in group I $(67 \%)$ had a prior history of cutaneous malignancy, versus 12 patients (52\%) in group II. Overall, 17\% of tumors in the control group $(n=4)$ were recurrences, versus $13 \%(n=3)$ in group II.

In the control group, $83 \%$ of lesions $(n=19)$ were BCCs, $13 \%(n=3)$ were SCCs, and $4 \%(n=1)$, metatypical carcinoma. In group II, BCCs accounted for $87 \%$ of 
all lesions $(n=20)$, and SCCs, for $13 \%(n=3)$. In both groups, the nose was the most common site of lesion (Graph 1 and 2).

The mean lesion size was $0.78(0.3-1.5) \mathrm{cm}$ (mode, $1 \mathrm{~cm}$ ) in group I and $1.18(0.4-3.0) \mathrm{cm}$ (mode, $0.5 \mathrm{~cm}$ ) in group II. The difference was statistically significant by Fisher's exact test $(\mathrm{p}=0.003)$.

Dermoscopic examination of lesions in the intervention group resulted in an increase in surgical margins in at least one area in all cases. Quantitatively, the increase in margins ranged from $0.5 \mathrm{~mm}$ to $7 \mathrm{~mm}$ (mean, $2.26 \mathrm{~mm}$; mode, $1 \mathrm{~mm}$ ).

In the control group, MMS was performed in a single stage in $57 \%$ of cases $(n=13)$ and in two stages in the remaining cases. In the intervention group, the surgery was completed in a single stage in $52 \%$ of cases $(n=12)$, in two stages in $35 \%(n=8)$, in three stages in $9 \%(n=2)$, and in four stages in $4 \%(n=1)$ (Graph 3 and 4). There were no significant betweengroup differences by the chi-square test $(p=0.399)$.

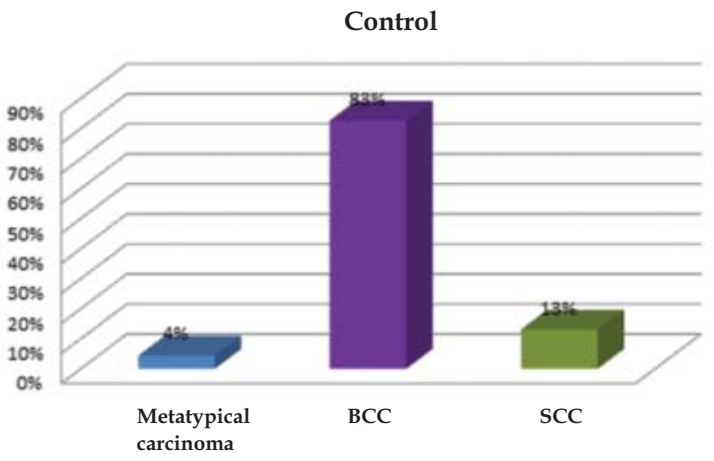

GRAPH 1: Distribution of histological types in the control group

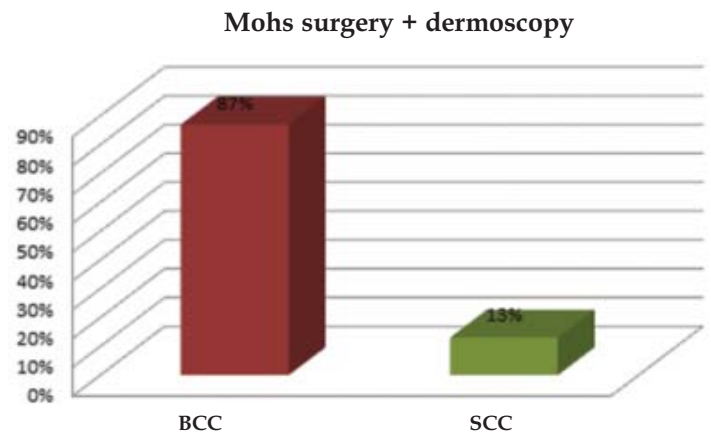

GRAPH 2: Distribution of histological types in the intervention group

\section{Control}
$1^{\text {st }}$ stage
$2^{\text {nd }}$ stage

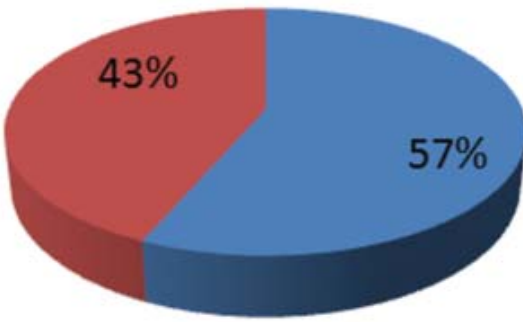

GRAPH 3:

Distribution of the number of surgical stages required in control group procedures

GRAPH 4:

Distribution of the number of surgical stages required in intervention group procedures

\section{DISCUSSION}

Mohs micrographic surgery (MMS) is the surgical technique associated with the highest cure rates and the lowest recurrence rates in the treatment of skin cancer, the most prevalent malignancy in the general population., $4,6,10,17,18$

In MMS, the tumor is excised and its peripheral and deep margins are assessed histologically ("micro") during the procedure, thus enabling a precise mapping ("graphic") of the lesion. Margins are examined by means of horizontal sections, rather than the standard vertical sections. ${ }^{4,6,11,19}$ MMS permits individualized treatment and identification of subclinical

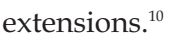

Historically, MMS was developed by Frederic Mohs at the University of Wisconsin in the 1930s and first described in 1941. It was initially known as chemosurgery due to the use of a zinc chloride-based paste for in vivo tissue fixation, a painful procedure that took 24 hours on average. The tumor was removed, sectioned, and stained, and all margins were examined microscopically during the procedure. If any tissue involvement was identified, the surgical margins were widened after an additional fixation cycle, 
on the basis of a map of the excised tissue. In 1953, the procedure was modified to employ fresh tissue histology for margin assessment, decreasing patient discomfort while producing equivalent outcomes, as proven by Tromovitch \& Stegman in 1974., 3,4,10,19

Currently, lesions are excised with a $2-3 \mathrm{~mm}$ margin. The tissue is then mapped to determine the location of each section for histological examination. The surgical specimen is frozen, cut into horizontal sections, and stained. The peripheral and deep margins are examined by the surgeon. If there is any remaining tumor tissue, another excision is performed, restricted to the site of the positive margin as marked on the surgical map. ${ }^{3,45,5,18,19}$ These steps are repeated until completely tumor-free margins are achieved.

MMS provides histological control of $100 \%$ of the surgical margin with maximal preservation of healthy adjacent tissue. ${ }^{4,6,16,17,18,19,20}$ This leads to greater preservation of important anatomic structures, making reconstruction simpler and safer, providing better cosmetic and functional results and reducing the risk of recurrence. ${ }^{4,6,10,17,18}$

The main histological types in which MMS is indicated are the aggressive subtypes of BCC (sclerodermiform, infiltrative, and micronodular), highgrade or invasive SCC, adnexal neoplasms, and dermatofibrosarcomas. Its use in melanoma is still contro-

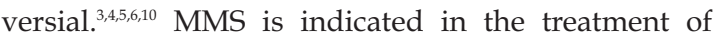
locally invasive cutaneous tumors, recurrent tumors or those with a high risk of recurrence, those with poorly defined borders, those larger than $2 \mathrm{~cm}$, lesions with perineural invasion, those located at sites where preservation of adjacent tissues is essential (eyelid, nose, extremities of the feet and hands, genitalia), and those located where the risk of recurrence is greatest, such as branchial cleft sites.

Despite its many benefits, MMS is still constrained by limitations regarding operative time and required infrastructure. ${ }^{6,10}$ Regarding cost, Cook et al showed that the cost of single-stage or two-stage MMS is equivalent to that of conventional surgery. ${ }^{3}$ However, due to the low rates of recurrence, there is less need for supplemental therapy and, consequently, lower additional costs. ${ }^{6}$

In an attempt to improve on MMS and minimize its restrictions, some techniques - such as curettage and photodynamic therapy - are sometimes used to demarcate tumor margins with greater precision. However, there is no standard protocol for determination of tumor extent prior to definition of surgical margins, an essential step for reduction of operative time. ${ }^{4,5}$

The present study assessed whether the use of dermoscopy for demarcation of surgical margins could improve the Mohs technique. A 10x-magnification dermoscope was chosen due to practical consid- erations, as this is the most widely used device in daily clinical practice, although it does not provide the wealth of detail obtained with 40x-magnification digital dermoscopes.

Dermoscopy, also known as dermatoscopy, epiluminescence microscopy, or surface microscopy, is a noninvasive technique that is very useful for assessment of skin lesions, as it enables visualization of structures invisible to the naked eye. ${ }^{5,7,8,12,13,14,15}$ It is the diagnostic tool that has had the single greatest clinical impact on the practice of dermatology. ${ }^{9}$ Dermoscopy enables in vivo assessment of tissues from the epidermis down to the reticular dermis, and thus represents an interface between clinical practice and histopathology. $5.7,8,12,13,14,20$

Dermoscopic assessment of skin lesions reduces the uncertainty of clinical examination, improving diagnostic accuracy and enabling detection of tumors at earlier stages. ${ }^{7,9,13,15,16,20,21}$ Furthermore, it prevents unnecessary biopsies and can provide guidance on the optimal site for biopsy, thus allowing the dermatopathologist to focus on the area of greatest clinical suspicion, which improves histopathological accuracy. ${ }^{7,20,21,22}$

First developed for assessment of pigmented lesions, dermoscopy is now employed in the examination of other cutaneous lesions, such as nonmelanoma skin cancers, angiomas, sebaceous hyperplasia, and hair and nail disorders. Use of dermoscopy for therapeutic monitoring of imiquimod treatment has also been reported. ${ }^{20,22}$

Caresana \& Giardini described the use of dermoscopy to assist in the determination of the peripheral borders of BCCs and reduce the surgical margin to $2 \mathrm{~mm}$ for conventional surgery, achieving a $98.5 \%$ rate of complete tumor excision. ${ }^{16}$ Another potential application is the enhancement of margin demarcation for MMS, as tested herein. ${ }^{5}$

Dermoscopy is a simple and practical technique whereby a dermoscope, a handheld device with 10x magnification, is used to examine the skin. In contact dermoscopy, the device emits a beam of light that strikes the skin at a $20^{\circ}$ angle. To eliminate reflections and thus enable better visualization of lesion structures, a fluid (oil, water gel, glycerin, or ethanol gel) is placed between the skin and the device.,12,14 Polarized light dermoscopes, which do not require the use of fluids and thus shorten the examination time, are another option. Despite their more practical nature, these dermoscopes can distort colors. Several of these devices also have contact dermoscopy capabilities in an attempt to minimize such color distortions..$^{12,14}$

Diagnostic criteria for assessment of pigmented lesions are well established, and several assessment methods are known, such as the ABCD rule, the 7-point checklist, the point rule, and Menzies' method. ${ }^{7,12,14}$

Diagnosis of pigmented BCC is based on the 
absence of a pigmented network and the presence of at least one of the following criteria: multiple bluegray globules; leaf-like areas; spoke-wheel areas; large, blue-gray ovoid nests; ulcerations; and arborizing telangiectasias. ${ }^{9}$ Concentric structures (early phase of spoke-wheel areas) and multiple "in focus" bluegray dots are considered non-classic patterns. ${ }^{9}$ Additionally, pigmented BCCs may also present with dermoscopic features commonly found in melanocytic lesions, such as brown-to-black dots or globules, veil-like structures, and pseudopods. The frequency at which these structures are found increases with the degree of lesion pigmentation. ${ }^{9}$

In non-pigmented BCC, particular attention should be paid to vascular structures. ${ }^{6,13}$ Whitish to erythematous, translucent to opaque areas free of vascular structures are characteristic. Multiple, small superficial ulcerations ( $\mathrm{n} \geq 5$ and $\leq 1 \mathrm{~mm}$ diameter) and fine, superficial telangiectasias are frequent. Arborizing vessels are the most specific finding, although not the most common one. . $9,13^{2}$

Vascular patterns play a very important role in the differential diagnosis of skin tumors. According to Argenziano et al, dotted vessels are also commonly found on dermoscopy, and are mostly associated with Spitz nevi. Linear-irregular vessels are common in melanoma, which can also feature dotted and atypical vessels. Congenital nevi are associated with comma vessels. Glomerular vessels are seen in Bowen disease. Crown vessels are seen in sebaceous hyperplasia. Finally, hairpin vessels are most closely correlated with seborrheic keratoses, but can also be observed in SCC. ${ }^{15}$ In addition to the aforementioned hairpin vessels, glomerular vessels amid areas of desquamation have also been reported in SCC. ${ }^{15,23}$ Criteria for dermoscopic diagnosis of some adnexal neoplasms have only been described in case reports. ${ }^{7}$

Superficial tumor-related telangiectasias are often difficult to distinguish on photodamaged skin with actinic lesions and severe poikiloderma (Figure 3). In addition, tumor margins may be difficult to demarcate in patients with biopsy or previous surgery scars (Figure 4) , $^{5,16}$

Dermoscopic analysis of lesion margins might mitigate this difficulty, but failed to do so in the present study. Possible explanations for the absence of significant differences between the control and intervention groups include the fact that, in lesions with overt clinical characteristics, the impact of dermoscopy is relatively minor, and therefore not associated with a change in the number of surgical stages of MMS. ${ }^{21}$ Furthermore, subclinical extensions are unpredictable and, although dermoscopy can guide excision of lateral margins, preoperative assessment of deep margins is impossible. ${ }^{16}$

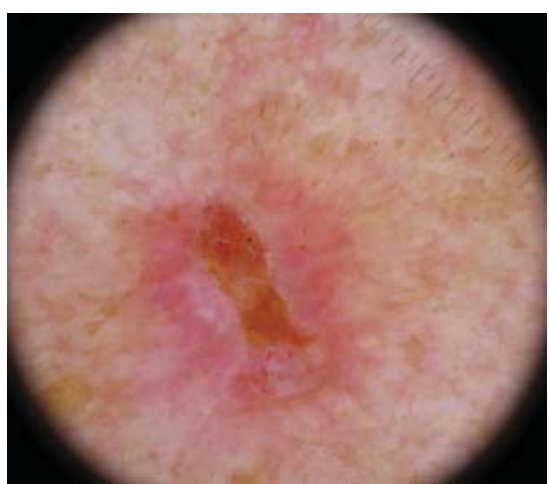

FIGURE 3: Telangiectasi as in photo$\mathrm{d}$ a $\mathrm{m}$ a g e d skin can be difficult to distinguish from tumor vessels

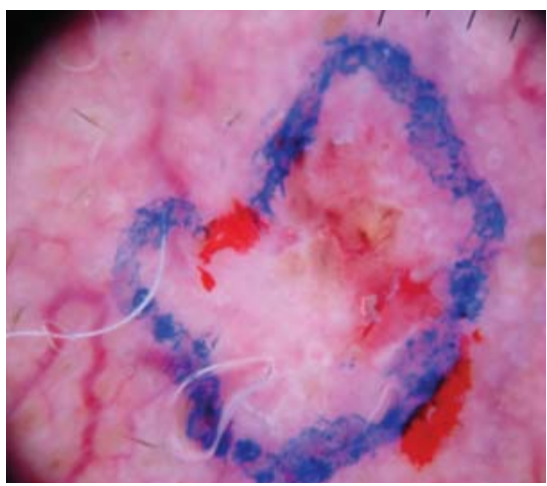

FIGURE 4:

Scars can hinder delimitation of lesion margins

As operator experience will have improved with each procedure, dermoscopic examination may have been conducted differently in the last few surgeries of the sample. As dermoscopy is operatordependent, assessment may be influenced not only by knowledge and experience but also by the time available for decision-making and by the dermatologist's self-confidence. ${ }^{21}$

\section{CONCLUSION}

Although outcomes were equivalent in the control and intervention groups, demonstrating that dermoscopy would have no impact on demarcation of surgical margins for MMS, this study puts forth a practical proposal for improvement of the Mohs technique. Nevertheless, studies with larger, population-based samples are still required to confirm these findings. $\square$

\section{ACKNOWLEDGEMENTS}

The authors would like to thank Fernanda Manfron Rosas, MD, Larissa L Gomes da Silva, MD, and Themis Hepp, MD, for their assistance. 


\section{REFERENCES}

1. Brasil. Ministério da Saúde. Instituto Nacional de Câncer. Estimativa 2010 - Incidência de câncer no Brasil. Rio de Janeiro: INCA; 2009. 98 p. [Acesso 22 Nov 2011] Disponível em: http://www.inca.gov.br/estimativa/2010/estimativa20091201.pdf

2. Sampaio SAP, Rivitti EA. Dermatologia. 3. edição. São Paulo: Editora Artes Médicas; 2007.

3. Galimberti G, Pontón Montaño A, Ferrario D, Kowalczuk A, Galimberti R. Mohs micrographic surgery for the treatment of basal cell carcinoma. Actas Dermosifiliogr. 2010 Dec;101:853-7.

4. Cumberland L, Dana A, Liegeois N. Mohs micrographic surgery for the management of nonmelanoma skin cancers. Facial Plast Surg Clin North Am. 2009;17:325-35.

5. Guardiano RA, Grande DJ. A direct comparison of visual inspection, curettage, and epiluminescence microscopy in determining tumor extent before the initial margins are determined for Mohs Micrographic Surgery. Dermatol Surg. 2010;36:1240-4.

6. Lang PG Jr. The role of Mohs' micrographic surgery in the management of skin cancer and perspective on the management of the surgical defect. Clin Plast Surg. 2004;31:5-31.

7. Massone C, Di Stefani A, Soyer HP. Dermoscopy for skin cancer detection. Curr Opin Oncol. 2005;17:147-53.

8. Scalvenzi M, Lembo S, Francia MG, Balato A. Dermoscopy patterns of superficial basal cell carcinoma. Int J Dermatol. 2008:47:1015-8.

9. Altamura D, Menzies SW, Argenziano G, Zalaudek I, Soyer HP, Sera F, et al. Dermatoscopy of basal cell carcinoma: Morphologic variability of global and local features and accuracy of diagnosis. J Am Acad Dermatol. 2010;62:67-75.

10. Terzian LR, Nogueira VMA, Paschoal FM, Barros JC, Machado-Filho CAS. Cirurgia micrográfica de Mohs para preservação tecidual nas cirurgias oncológicas da face. Surg Cosmet Dermatol. 2010;2:257-63.

11. Mosterd K, Krekels GA, Nieman FH, Ostertag JU, Essers BA, Dirksen CD, et al. Surgical excision versus Mohs' micrographic surgery for primary and recurrent basal-cell carcinoma of the face: a prospective randomized controlled trial with 5-years' follow-up. Lancet Oncol. 2008;9:1149-56.

12. Campos-do-Carmo G, Ramos-e-Silva M. Dermoscopy: basic concepts. Int J Dermatol. 2008;47:712-9.

13. Giacomel J, Zalaudek I.. Dermoscopy of superficial basal cell carcinoma. Dermatol Surg. 2005;31:1710-3

14. Frange VMN, Arruda LHF, Daldon PEC. Dermatoscopia: importância para a prática clínica. Rev Ciênc Méd. 2009;18:209-15.

15. Argenziano G, Zalaudek I, Corona R, Sera F, Cicale L, Petrillo G, et al. Vascular structures in skin tumors: A dermoscopy study. Arch Dermatol. 2004;140:1485-9.

16. Caresana G, Giardini R. Dermoscopy-guided surgery in basal cell carcinoma. J Eur Acad Dermatol Venereol. 2010;24:1395-9

17. Cook J, Zitelli JA. Mohs micrographic surgery: A cost analysis. J Am Acad Dermatol. 1998:39:698-703.

18. Terzian LR, Festa-Neto C, Pimentel, ERA. Predictors of higher number of stages in Mohs Micrographic Surgery for the treatment of Squamous Cell Carcinoma of the Head. An Bras Dermatol. 2008;83:221-6.

19. Smeets NW, Kuijpers DI, Nelemans P, Ostertag JU, Verhaegh ME, Krekels GA, et al. Mohs' micrographic surgery for treatment of basal cell carcinoma of the face - results of a retrospective study and review of the literature. Br J Dermatol. 2004;151:141-7.

20. Terushkin V, Wang SQ. Mohs surgery for basal cell carcinoma assisted by dermoscopy: Report of two cases. Dermatol Surg. 2009;35:2031-5.

21. Benvenuto-Andrade C, Dusza SW, Hay JL, Agero AL, Halpern AC, Kopf AW et al. Leve of confidence in diagnosis: Clinical examination versus dermoscopy examination. Dermatol Surg. 2006;32:738-44.

22. de Troya-Martín M, Frieyro-Elicegui M, Fúnez Liébana R, Aguilar Bernier M, FernándezCanedo NI, Blázquez Sánchez N. Lentigo maligna managed with topical imiquimod and dermoscopy: report of two cases. Dermatol Surg. 2008;34:1561-6.

23. Felder S, Rabinovitz H, Oliviero M, Kopf A. Dermoscopy differentiation of a superficial basal cell carcinoma and squamous cell carcinoma in situ. Dermatol Surg. 2006;32:423-5.

\author{
MAILING ADDRESS: \\ Heliane Sanae Suzuki \\ Rua General Carneiro, 181 SAM 4 \\ 80060-900 - Curitiba -PR \\ Brazil \\ E-mail:helianesuzuki@yahoo.com.br
}

How to cite this article: Suzuki HS, Serafini SZ, Sato MS. Utility of dermoscopy for demarcation of surgical margins in Mohs micrographic surgery. An Bras Dermatol. 2014;89(1):38-43. 